\title{
Applying Cardiac Rehabilitation Exercise Protocol to Reduce Post-operative Cardio-Pulmonary Complications among Open Heart Surgery Patient's
}

\author{
${ }^{1}$ Assistant Lecturer of Critical Care Nursing, Faculty of Nursing-Minia University. \\ ${ }^{2}$ Prof .dr. of Medical Surgical Nursing. Faculty of Nursing- Ain-Shams university \\ ${ }^{3}$ Assistant Prof. Cardiothoracic Surgery, Faculty of Medicine- Minia university \\ ${ }^{4}$ Assistant prof. of Medical Surgical Nursing, Faculty of Nursing - Minia University
}

Ahmed A. A. ${ }^{1}$ Hassan M. S. ${ }^{2}$ Mohamed Y. S. ${ }^{3}$ Gamal L. M. ${ }^{4}$

\begin{abstract}
Background: Cardiac surgery is a complex procedure that is resulting in a higher incidence of complications and tends to significantly decrease the potential for recovery. It is essential for the nurse to anticipate the treatment of complications so that appropriate interventions are initiated in order to ensure a positive outcome for patients. Aim of the Study: To evaluate the effect of applying cardiac rehabilitation exercise protocol for open heart surgery patient's to reduce post-operative cardio-pulmonary complications. Research Design: Quasi-experimental research design was utilized in the current study. Subjects: A purposive sample including 60 patients male and female, classified equally into two equal groups; study group $(n=30)$ and control group $(n=30)$. Setting: This study carried out in the inpatient cardiac surgery department of Cardio-thoracic Surgery Hospital at New Minia City. Tools of Data Collection: Three tools were utilized in collecting data. Tool I: Patient Assessment Sheet, Tool II: Cardiac Rehabilitation Exercise Checklist. Tool Ш: Cardiopulmonary Complications Assessment Sheet. Results: Current study findings revealed that the study group had a lower incidence of cardiac and respiratory complications $(30 \%$, and $3.3 \%)$ respectively compared to $(46.7 \%$ and $26.7 \%)$ for the control group and there is a statistical significance differences were detected between the two groups. Conclusion: The current study findings concluded that the cardiac rehabilitation exercise protocol in the form of deep breathing, incentive spirometer, coughing and early ambulation induces significant improvement in reducing the risk of post-operative cardio pulmonary complications. Recommendations: Hospitals should recommend implementing cardiac rehabilitation exercise protocols as a routine hospital policy among all cardiac surgical patients in all age groups.
\end{abstract}

Keywords: Open Heart surgery, Postoperative Cardiac and Pulmonary Complications, Rehabilitation Exercise Protocol.

\section{Introduction}

Cardiac surgery is a well-established procedure worldwide with excellent results for efficacy and safety in treating heart patients. Cardiac surgery, including coronary artery bypass, valve surgery, and congenital defect repair, is among the most common classes of surgical procedures performed worldwide. Successful outcomes after cardiac surgery depend on optimum postoperative critical care. (Stephens \& Whitman, 2015) \& (da Costa Torres, 2016)

The incidence of postoperative complications after cardiac surgery is reported according to the specific complication, presence of comorbidities, and patient related factors (e.g., age). Factors such as age, duration of extracorporeal circulation (ECC), and respiratory complications (including atelectasis, pleural effusion, and respiratory failure) may increase the length of hospital stay. (Ettema, et al., 2014 \& Cordeiro, et al., 2017)

Major complications include cardiac, respiratory, and renal dysfunction, as well as gastrointestinal and neurological complications. The pathogenesis behind postoperative complications involves hemodynamic and inflammatory signaling pathways, which activated in response to cardiac arrest, artificial and non-pulsatile circulation, hypothermia, blood contact with the artificial surface of cardiopulmonary bypass (CPB) and the surgical insult with skin incision and sternotomy. (Enger, 2017)

In surgery, it is important for patients to go into the theatre fully prepared physically and emotionally regarding the procedures they are about to go through. Surgical patients go through either planned (elective) or emergency surgery. In planned surgeries, patients go through a multidisciplinary team of experts' examination depending on the patient's $P$ a g e $\mid 45$ condition. Some of these experts include the nurse, the anesthetist, the surgeon, the clinical psychologist, laboratory technician and others. (Adugbire, et al., F. 2017)

Patients recovering from cardiac surgery may be referred to a cardiac rehabilitation (CR) program. The aim of $\mathrm{CR}$ is to increase survival, reduce cardiovascular disease (CVD)-related morbidity and hospital admissions, improve functional capacity, quality of life and facilitate early return to work. Structured exercise training is one of the essential components of cardiac rehabilitation and may make the largest contribution to increasing patient survival. (Nichols, et al., 2018)

After cardiac surgery, inpatient cardiac rehabilitation (ICR) contributes significantly to the prevention of lung complication and decondition caused by prolonged bed rest, thus to early recovery. Exercise therapy is the essential component of ICR which composed of breathing exercises, early mobilization, and strength exercises therapy. (Seo, et al., 2017)

The cardiac rehabilitation exercise program during hospitalization consists of respiratory physiotherapy, neck and shoulder exercises, walking and cycling. The purpose is to prevent respiratory complications such as atelectasis and pneumonia, neck and shoulder pain and increasing the physical functional level. (Højskov, et al., 2017)

It is important that nurses educate surgical patients on the importance of deep breathing and coughing, regular gentle leg exercise and early mobilization to reduce the risk of postoperative complications (Snowdon, et al., 2014). 


\section{Significance of the Study:}

The documented incidence of post-operative complications ranges from 3\% to $16 \%$ after Coronary Artery Bypass Graft and 5\%-7\% after valvular heart surgery. (Naveed, et al., 2017) The cumulative incidence of pulmonary complications was $15.08 \%$ with a mortality rate of $18.5 \%$ from patients who developed complications and overall mortality among all patients was $2.79 \%$. Pulmonary complications occurred in $7.82 \%$ of patients with coronary artery revascularization, $2.23 \%$ in patients with valvular replacement and $5.05 \%$ in patients with congenital heart disease. (Al-Qubati, Damag \& Noman, 2013)

\section{Aim of the Study}

The aim of the present study was to evaluate the effect of applying cardiac rehabilitation exercise protocol for open heart surgery patient's to reduce post-operative cardiopulmonary complications

\section{Research Hypothesis}

To fulfill the aim of the study, It is hypothesized that the patients undergoing open heart surgery who will be exposed to cardiac rehabilitation exercises (study group) will have a lower incidence of cardio-pulmonary complications compared to the (control group).

\section{Subjects and Methods \\ Research Design}

Quasi-experimental research design was utilized in the current study.

\section{Setting:-}

This study carried out in the inpatient cardiac surgery department of Cardio-thoracic Surgery Hospital at New Minia City.

\section{Subjects:}

A purposive sample of 60 patients was collected through fourteen (14) month and they were classified equally into two groups; $(\mathrm{n}=30)$ for control group and $(\mathrm{n}=30)$ for study group who were willing to participate in the current study. Both groups in the current study were selected according to the following inclusion and exclusion criteria:

\section{Inclusion Criteria:-}

- All adult (18-60 years) patients admitted in cardiac surgery department two days before open heart surgery

- Hemodynamically stable

- Conscious

\section{Exclusion Criteria:-}

- Patients undergoing invasive or noninvasive mechanical ventilation for a period exceeding 24 hours after admission to the Intensive Care unit (ICU).

- Patients contraindicated to perform the exercise

- Any complications other than cardiopulmonary complications

\section{Tools of Data Collection:-}

- The current study data was collected by using three tools that were developed by the investigator after revising extensive relevant literature review and reviewed by a panel of nine experts, fourth in the field of Medical Surgical Nursing Faculty of Nursing Ain- Shams University and three in the field of Medical Surgical Nursing Faculty of Nursing Minia University and two in the field of cardiac surgery Faculty of Medicine at Minia University for validity testing. These tools are as following:-

First Tool: Health Assessment Sheet: It includes two Parts:

- $\mathbf{1}^{\text {st }}$ Part: Socio- demographic data as name, age, gender, level of education and marital status, occupation, income and phone number.

- $2^{\text {nd }}$ Part: Medical data, based on (Lewis, et al., 2014) as: medical diagnosis, type of surgery, presence of chronic diseases, smoking and body mass index (BMI), hemodynamic variables (Temperature, Heart Rate, Blood pressure, Respiration, and Oxygen Saturation)

Second Tool: Cardiac Rehabilitation Exercise Checklist which includes two Parts: adopted from (García, et al, 2014)

- $\quad \mathbf{1}^{\text {st }}$ Part: Respiratory Physiotherapy consisted of 11 step (Incentive Spirometer include 3 steps, Deep Breathing include 4 steps and Breath and Cough include 4 steps)

- $\quad 2^{\text {nd }}$ Part: Upper and Lower Limb Exercises consisted of 58 step (upper and lower limb ROM include 48 step, walking include 3 steps and sternal precautions include 7 steps)

- They were applied to reduce the postoperative cardio pulmonary complications among open heart surgery patients.

- Scoring system: The maximum score value was 69 (respiratory physiotherapy 11 point and upper \& lower limb exercises 58 point), each item was observed, categorized, and scored into either done $=1$ or not done $=0$ on all items of the checklist. These scores are further classified as unsatisfactory level (less than 60\%), and satisfactory level more than $60 \%)$.

Third Tool: Cardiopulmonary Complications Assessment Sheet based on (Kashmiri, et al., 2012):

- This tool used to evaluate the effect of the applied cardiac rehabilitation exercise protocol on the occurrence of postoperative cardiac and pulmonary complications among open heart surgery patients.

- The investigator assessed the patient for presence of cardiac complications as (Impaired Cardiac output syndrome, Cardiac Arrhythmias) or pulmonary complications (Respiratory tract infection, Pneumothorax, Hemothorax or ARDS) before discharge (after applying the cardiac rehabilitation exercise protocol).

Tools Validity:-

Content validity was done to identify the degree to which the used tools measure what was supposed to be measured. The developed tools were examined by a panel of nine expert's opinion in the field of the study, one assistant professor and two lecturers of Medical Surgical Nursing at Minia University - Faculty of Nursing and two assistance professors of cardiac surgery Department Faculty of Medicine 
at Minia University and three professors and one assistant professor of Medical Surgical Nursing at Ain- Shams University and according to their opinion some modifications were applied.

\section{Tools Reliability:-}

Reliability was ascertained statistically by using Alpha Cronbach test to ensure that the study tools are reliable. Reliability of Health Assessment sheet, Cardiac Rehabilitation Exercise Checklist and Cardiopulmonary Complications Assessment Sheet were $(0.96,0.71$ and 0.86$)$ respectively.

\section{Pilot Study:-}

A pilot study was carried out on $10 \%(n=6)$ of the total sample to test the clarity of tools and estimate the time required for fulfilling it. Based on results of the pilot study some modifications were done and the subjects were not included to the actual sample.

\section{Ethical Considerations:-}

An official permission to conduct the study was obtained from the Ethical committee in the Faculty of Nursing, Dean of Faculty of Nursing, and Manager of Cardiothoracic Minia University Hospital, and agreement from Egypt Academic for Research Center and Technology to carry out this study. Subject's participant in this study were voluntary and each involved subject were informed about the purpose, procedure, benefits, and nature of the study, and that he/she had the right to withdraw from the study at any time without any rationale, then written consents were obtained. Confidentiality and anonymity of each subject were ensured through coding of all data and protecting the obtained data.

Educational Training Practices about the Cardiac Rehabilitation Exercise Protocol formulated by the investigator after revising extensive relevant literature review such as (García, et al., 2014) \& (Højskov, et al., 2017). They were applied to reduce the postoperative cardiopulmonary complications among open heart surgery patients. Arabic booklet was included which covered; Introduction of the Cardiac Surgery, Importance of Exercises, and Complete Description of Each Exercise (deep breathing exercise include 4 steps, deep breath and cough include 4 steps and Incentive spirometer include 3 steps, upper and lower limb range of motion include 48 step, walking include 3 steps and sternal precautions 7 steps)

The investigator had trained the study subjects about the educational cardiac rehabilitation exercise booklet in the preoperative phase to ensure the subjects ability to perform these exercises postoperatively.

The investigator asked the participants to perform breathing exercises (7-10 breaths and incentive spirometer 3$5 \mathrm{~min}) 4$ times a day. Physical exercises include shoulder and head exercises, resistance training, leg endurance and walking (10 sessions of each exercise two times per day from postoperative day 1 until hospital discharge.

\section{Procedure:-}

\section{Preparatory Phase:}

(Assessment and planning phase) It included reviewing of the current and relevant related literature and theoretical knowledge of the various related aspects using books, articles, internet, and periodical magazines in order to develop the data collection tools. Exploring prevalence of patient at cardiac surgical ward and incidence of cardiopulmonary complications among those patient's

\section{Implementation Phase:}

This phase includes the preoperative and postoperative period and during this phase the cardiac rehabilitation exercise training protocol was implemented.

\section{Pre-operatively:}

Selected subjects (according to inclusion and exclusion criteria) were admitted to inpatient cardiac surgery department, Patients were equally enrolled in this study as control and study groups. The investigator obtained written consent from those (study and control group) who accepted to participate in this study. For the study group, patients who were scheduled for the cardiac rehabilitation exercise protocol were informed by the investigator individually about purpose and nature of the study

The investigator has started data collection from control group firstly by using the first and second tools (Health assessment sheet and the cardiac rehabilitation exercise checklist to assess the patients baseline data about the cardiac rehabilitation exercises) these data was collected by the investigator preoperatively for only one time.

Preoperative training about the educational cardiac rehabilitation exercise booklet over a period of one or two days was done by the investigator to each participant for the study group through face to face interview to follow the prescribed instructions. The total number of sessions for data collection \& training of the study group patient was 2 - 3 sessions preoperatively varied according to each participant understanding. Duration for each session was ranged from 30 to 45 minutes.

The first session (overview about cardiac surgery); at the beginning of this session, the investigator introduce herself and explained the objective of the research; each study group patients was given an educational cardiac rehabilitation exercise booklet, the investigator illustrated the content of the booklet, give the patients simple information about anatomy of the heart, physiology of the heart, cardiac surgery, and preoperative routine events.

The second session (Pre and postoperative exercises); the investigator gave the patients a detailed explanation about each exercise and help the patients develop skills related to respiratory physiotherapy (deep breathing, coughing exercise and incentive spirometer), change positioning, ambulation and extremities exercises and also instructions regarding duration and frequency of each previous skill.

Demonstration and re-demonstration was done to ensure that each patient perform these exercises accurately. On the other hand; the investigator trained the nursing staff in the cardiac surgery department as co- investigator who had shift in the same study setting to follow up the study group who was applied the cardiac rehabilitation exercise protocol in accurate time during their specific work shift.

\section{Postoperatively:}

The investigator assessed adult patients undergoing open heart surgery through daily basis from surgery day until discharge during morning and evening shift at inpatient cardiac surgery department. The assessment tools were collected from control group firstly then started the collection for other study group. 


\section{Evaluation Phase:}

The last phase of proposed teaching program is the evaluation phase. The investigator assessed both study and control group by using the second tool to evaluate the patient's performance of cardiac exercises after applying the cardiac rehabilitation exercise protocol (before discharge).

The investigator assessed patients for the presence of any cardiac or pulmonary complications using the third tool. (Cardiopulmonary Complications Assessment Sheet) The investigator assessed the presence of complications before the patient discharge from hospital signs and symptoms of complications was reported by the investigator after being observed by two physicians to confirm the absence of interobserver variability.

\section{Statistical Analysis of Data:}

Data were summarized, tabulated, and presented using descriptive statistics in the form of frequency distribution, percentages, means and the standard deviations as a measure of dispersion. A statistical package for the social science (SPSS), version (20) was used for statistical analysis of the data, as it contains the test of significance given in standard statistical books. Numerical data were expressed as mean \& SD. Qualitative data were expressed as frequency and percentage. Probability (P-value) is the degree of significance, less than 0.05 was considered significant. The smaller the Pvalue obtained, the more significant is the result $(*)$, less than 0.001 was considered highly significant $(* *)$ and correlation coefficient was done by using Pearson correlation test.

\section{Results}

Table (1): Distribution of Both Study and Control Groups Regarding to Their Socio-demographic Data (n=60)

\begin{tabular}{|c|c|c|c|c|c|c|}
\hline \multirow[t]{2}{*}{ Socio Demographic Data } & \multicolumn{2}{|c|}{ Study $(n=30)$} & \multicolumn{2}{|c|}{ Control $(n=30)$} & \multirow{2}{*}{$\begin{array}{c}\text { Significance } \\
\text { test }\end{array}$} & \multirow{2}{*}{ P-value } \\
\hline & No. & $\%$ & No. & $\%$ & & \\
\hline \multicolumn{7}{|c|}{ Age/ Years } \\
\hline $18->30$ & 7 & 23.3 & 3 & 10 & \multirow{5}{*}{$\mathrm{t}=1.36$} & \multirow{5}{*}{$\begin{array}{c}0.179 \\
\text { NS }\end{array}$} \\
\hline $30->40$ & 8 & 26.7 & 9 & 30 & & \\
\hline $40->50$ & 8 & 26.7 & 8 & 26.7 & & \\
\hline$\leq 50$ & 7 & 23.3 & 10 & 33.3 & & \\
\hline Mean \pm SD & \multicolumn{2}{|c|}{$40.7 \pm 12.3$} & \multicolumn{2}{|c|}{$44.9 \pm 11.5$} & & \\
\hline \multicolumn{7}{|c|}{ Gender } \\
\hline Male & 11 & 36.7 & 13 & 43.3 & \multirow{2}{*}{$\chi^{2}=0.278$} & \multirow{2}{*}{$\begin{array}{c}0.598 \\
\text { NS }\end{array}$} \\
\hline Female & 19 & 63.3 & 17 & 56.7 & & \\
\hline \multicolumn{7}{|c|}{ Occupation } \\
\hline Employer & 15 & 50 & 14 & 46.7 & \multirow{2}{*}{$\chi^{2}=0.067$} & \multirow{2}{*}{$\begin{array}{c}0.796 \\
\text { NS }\end{array}$} \\
\hline Not Employer & 15 & 50 & 16 & 53.3 & & \\
\hline \multicolumn{7}{|c|}{ Level of Education } \\
\hline Illiterate & 10 & 33.3 & 9 & 30 & \multirow{5}{*}{$\chi^{2}=1.096$} & \multirow{5}{*}{$\begin{array}{l}0.895 \\
\text { NS }\end{array}$} \\
\hline Read \& write & 8 & 26.7 & 6 & 20 & & \\
\hline Primary & 5 & 16.7 & 6 & 20 & & \\
\hline Secondary & 5 & 16.7 & 5 & 16.7 & & \\
\hline Bachelor & 2 & 6.7 & 4 & 13.3 & & \\
\hline \multicolumn{7}{|c|}{ Marital Status } \\
\hline Single & 4 & 13.3 & 7 & 23.3 & \multirow{3}{*}{$\chi^{2}=3.01$} & \multirow{3}{*}{$\begin{array}{c}0.390 \\
\text { NS }\end{array}$} \\
\hline Married & 25 & 83.3 & 22 & 73.3 & & \\
\hline Divorced & 1 & 3.3 & 1 & 3.3 & & \\
\hline
\end{tabular}

\section{NS= Not Significant}

Table (1): Reveals distribution of the studied subjects according to the Socio-demographic data. In relation to age, the mean age among study and control groups was nearly similar $(40.7 \pm 12.3$ and $44.9 \pm 11.5)$ respectively. Regarding to gender it was found that the highest percentages among study and control group were female $(63.3 \%$, and $56.7 \%)$ respectively. Regarding occupation; it was seen that the highest percentages among study and control group were employer (50\% and $46.7 \%)$ respectively.

In relation to education levels it was found that the largest percent, $(33.3 \%, 30 \%)$ of study and control groups respectively were illiterate. Concerning marital status, the finding represented that the highest percentage among study and control group were married $(83.3 \%, 73.3 \%)$ respectively. There was no statistical significance difference between the two groups regarding to sociodemographic data at $(\mathrm{P} \leq 0.05)$

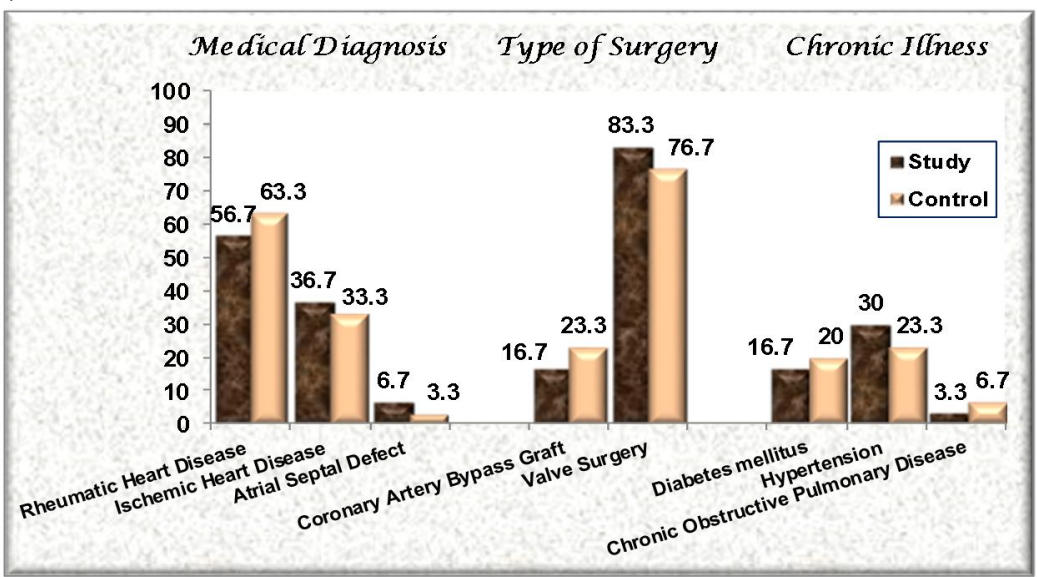

Figure (1): Distribution of both Study and Control Group Regarding to Medical Data 
Figure (1) Shows that the highest percentages of both groups (study and control) were having rheumatic heart disease $(56.7 \%$ \& $63.3 \%)$ respectively. As regarding type of surgery; it was found that the highest percentage of both groups (study and control) had valve surgery $(83.3 \%$ \& $76.7 \%)$ respectively. Regarding presence of chronic illness; it was observed that the highest percentage of both groups (study and control) had hypertension (30\% and $23.3 \%)$ respectively, while $(16.7 \%$ and $20 \%)$ had diabetes mellitus respectively.

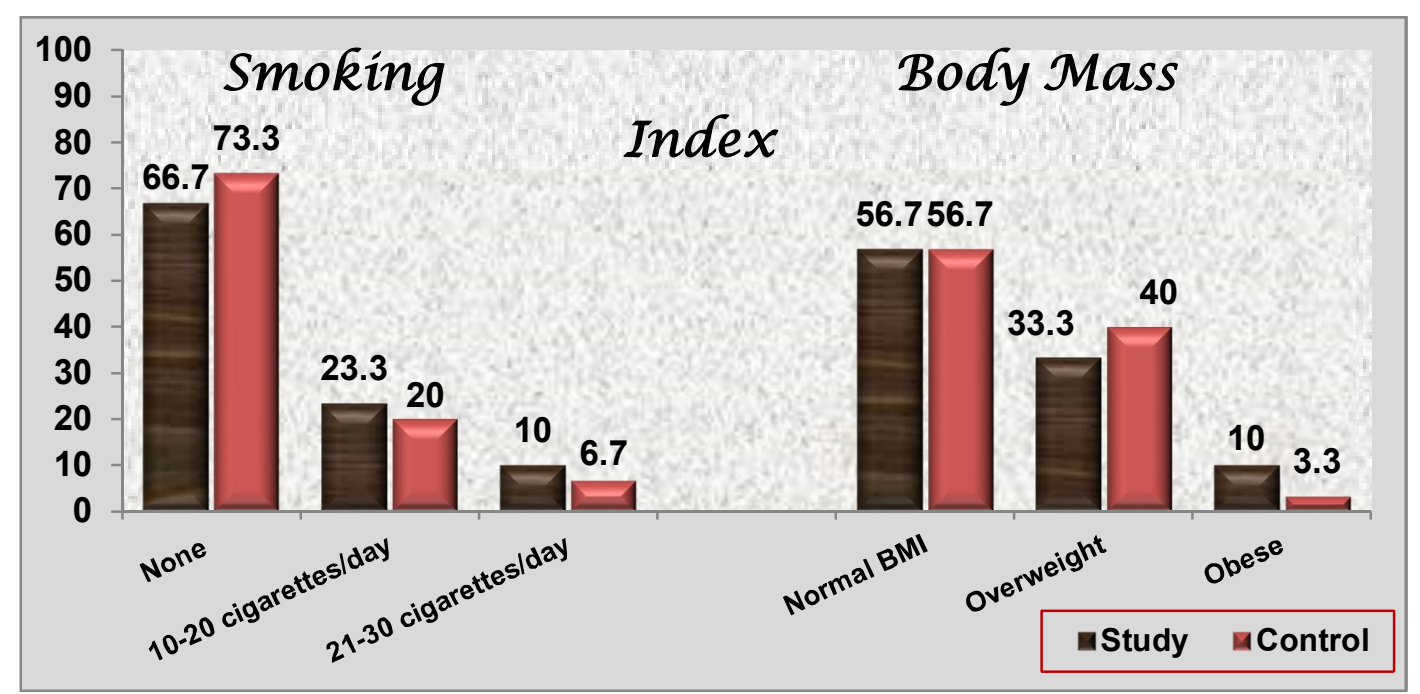

Figure (2): Distribution of both Study and Control Group Regarding to Smoking and BMI

Figure (2) Shows that the highest percentage of both groups (study and control) were none smoker $(66.7 \%$ and $73.3 \%$ ) respectively, while $(23.3 \%$ and $20 \%)$ of them respectively were smoke $10-20$ cigarettes/day. Regarding to BMI it was found that the highest percentage of both groups (study and control) were in the normal BMI score (56.7) for each group.

Table (2): Distribution of Study and Control Group Regarding Level of Performance Pre and Post Implementing Cardiac Rehabilitation Exercise Protocol $(n=60)$

\begin{tabular}{|c|c|c|c|c|c|}
\hline \multirow{2}{*}{ Exercise Protocol Score } & \multicolumn{2}{|c|}{ Study $(n=30)$} & \multicolumn{2}{|c|}{ Control $(n=30)$} & \multirow{2}{*}{ p value } \\
\hline & No. & $\%$ & No. & $\%$ & \\
\hline \multicolumn{6}{|l|}{ Pre-Intervention } \\
\hline - $\quad$ Satisfactory & $\mathbf{0}$ & $\mathbf{0}$ & $\mathbf{0}$ & $\mathbf{0}$ & \multirow[t]{2}{*}{0.248} \\
\hline - $\quad$ Unsatisfactory & 30 & 100 & 30 & 100 & \\
\hline \multicolumn{6}{|l|}{ Post- Intervention } \\
\hline Satisfactory & 27 & 90 & $\mathbf{0}$ & $\mathbf{0}$ & \multirow[t]{2}{*}{$0.000 * *$} \\
\hline Unsatisfactory & 3 & 10 & 30 & 100 & \\
\hline p value $^{2}$ & \multicolumn{2}{|c|}{$0.000 * *$} & \multicolumn{2}{|c|}{0.803} & \\
\hline
\end{tabular}

\section{** Highly Statistical Significant Difference $(\mathbf{P} \leq \mathbf{0 . 0 1})$}

Table (2) Reflected that there were no statistical significant differences between study and control group regarding performance of the cardiac rehabilitation exercise protocol pre intervention. While the findings showed a highly significant improvement between the study and control group post intervention (after implementing the cardiac rehabilitation exercise protocol) as documented by $\mathrm{p}$ value $(0.000)$

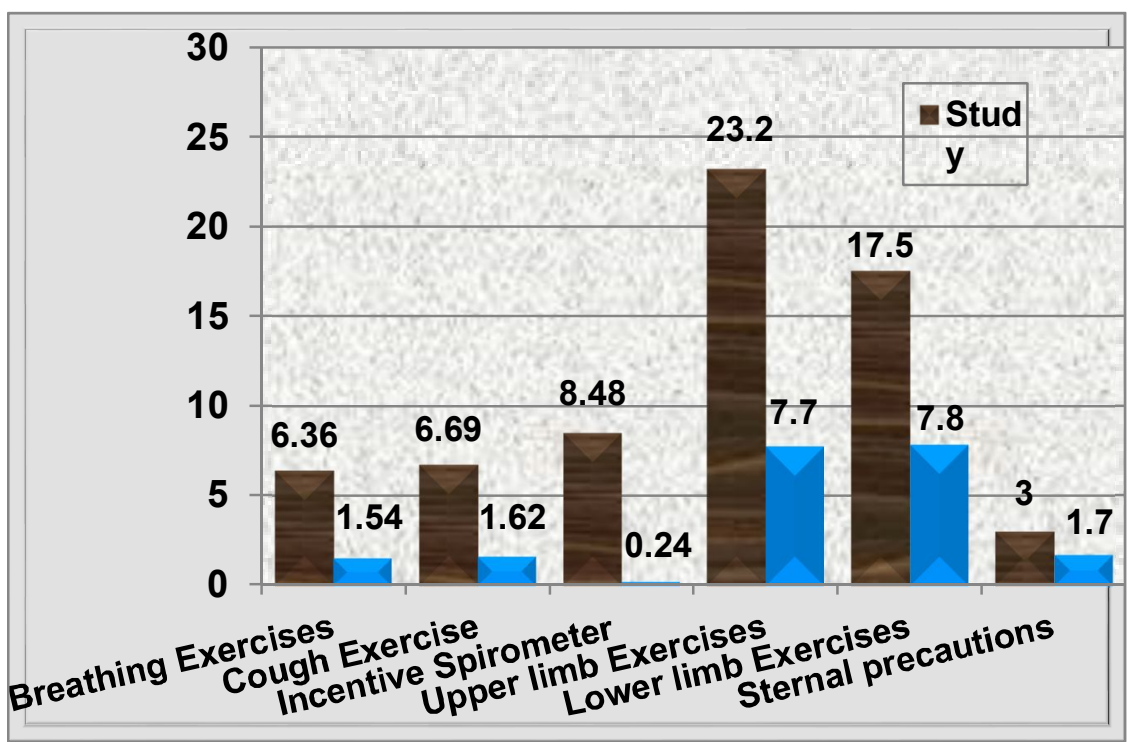

Figure (3): Mean Score of Study and Control Group Regarding Level of Performance Post Implementing Cardiac Rehabilitation Exercise Protocol $(n=60)$ 
Minia Scientific Nursing Journal (Print) (ISSN 2537-012X) Vol. (6) No. (1) December 2019

Figure (3) Reflected that the mean score of all items regarding each exercise level of performance was increased in study group compared to control group post implementing cardiac rehabilitation exercise protocol. Moreover a highly statistical significant difference was found as documented by p values $(0.000)$ for all items sores.

Table (3): Mean Score of Study and Control Groups Regarding to Their hemodynamic variables Pre and Post Implementing the Cardiac Rehabilitation Exercise Protocol $(n=60)$

\begin{tabular}{|c|c|c|c|c|}
\hline \multirow{2}{*}{\multicolumn{2}{|c|}{ Hemodynamic Variables }} & \multirow{3}{*}{$\begin{array}{c}\text { Study }(\mathbf{n}=\mathbf{3 0}) \\
\text { Mean } \pm \text { SD } \\
37.1 \pm 0.36 \\
\end{array}$} & \multirow{3}{*}{$\begin{array}{c}\text { Control }(\mathbf{n}=\mathbf{3 0}) \\
\text { Mean } \pm \text { SD } \\
36.9 \pm 0.15 \\
\end{array}$} & \multirow{3}{*}{$\begin{array}{c}\text { P-value }^{1} \\
0.09 \\
\end{array}$} \\
\hline & & & & \\
\hline \multirow{5}{*}{ 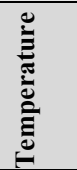 } & $1^{\text {st }}$ day & & & \\
\hline & $2^{\text {nd }}$ day & $37 \pm 0.20$ & $37 \pm 0.11$ & 0.219 \\
\hline & $3^{\text {rd }}$ day & $37.2 \pm 0.27$ & $37 \pm 0.11$ & 0.140 \\
\hline & $4^{\text {th }}$ day & $37 \pm 0.21$ & $37.2 \pm 0.27$ & $0.004 * *$ \\
\hline & $5^{\text {th }}$ day & $37 \pm 0.18$ & $36.9 \pm 0.11$ & $0.006^{* *}$ \\
\hline \multicolumn{2}{|c|}{ P-value $^{2}$} & \multicolumn{3}{|c|}{$(0.017 *)$} \\
\hline \multirow{5}{*}{ 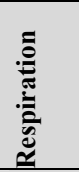 } & $1^{\text {st }}$ day & $15.3 \pm 0.96$ & $15 \pm 1.5$ & 0.379 \\
\hline & $2^{\text {nd }}$ day & $16.8 \pm 1.2$ & $18.4 \pm 2.2$ & $0.001 * *$ \\
\hline & $3^{\text {rd }}$ day & $15.1 \pm 0.75$ & $16.3 \pm 3.1$ & $0.049 *$ \\
\hline & $4^{\text {th }}$ day & $15.2 \pm 0.76$ & $16.2 \pm 3.04$ & $0.050 *$ \\
\hline & $5^{\text {th }}$ day & $15.03 \pm 0.66$ & $16.3 \pm 3.10$ & $0.029 *$ \\
\hline \multicolumn{2}{|c|}{ P-value ${ }^{2}$} & \multicolumn{3}{|c|}{$(0.000 * *)$} \\
\hline \multirow{5}{*}{$\stackrel{\mathscr{E}}{\Xi}$} & $1^{\text {st }}$ day & $86.1 \pm 4.6$ & $85.8 \pm 6.5$ & 0.827 \\
\hline & $2^{\text {nd }}$ day & $85.1 \pm 4.04$ & $82.5 \pm 3.8$ & $0.013^{*}$ \\
\hline & $3^{\text {rd }}$ day & $85.1 \pm 4.04$ & $82.6 \pm 4.4$ & $0.029 *$ \\
\hline & $4^{\text {th }}$ day & $84.9 \pm 3.9$ & $82.4 \pm 4.04$ & $0.021^{*}$ \\
\hline & $5^{\text {th }}$ day & $85 \pm 4.04$ & $82.5 \pm 4.1$ & 0.024* \\
\hline \multicolumn{2}{|c|}{ P-value $^{2}$} & \multicolumn{3}{|c|}{$(0.001 * *)$} \\
\hline \multirow{5}{*}{ 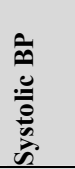 } & $1^{\text {st }}$ day & $134.8 \pm 6.5$ & $138.1 \pm 7.01$ & 0.712 \\
\hline & $2^{\text {nd }}$ day & $133 \pm 7.5$ & $132 \pm 7.13$ & 0.705 \\
\hline & $3^{\text {rd }}$ day & $129.2 \pm 5.5$ & $128.6 \pm 7.9$ & 0.746 \\
\hline & $4^{\text {th }}$ day & $128.8 \pm 5.4$ & $128.2 \pm 8.1$ & 0.757 \\
\hline & $5^{\text {th }}$ day & $128.8 \pm 5.4$ & $128.5 \pm 7.9$ & 0.895 \\
\hline \multicolumn{2}{|c|}{ P-value ${ }^{2}$} & \multicolumn{3}{|c|}{$(0.000 * *)$} \\
\hline \multirow{5}{*}{ 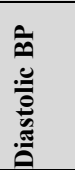 } & $1^{\text {st }}$ day & $78.2 \pm 8.5$ & $72.6 \pm 9.8$ & 0.069 \\
\hline & $2^{\text {nd }}$ day & $74.7 \pm 6.5$ & $76.8 \pm 7.01$ & 0.242 \\
\hline & $3^{\text {rd }}$ day & $80.5 \pm 3.9$ & $82.4 \pm 5.3$ & 0.115 \\
\hline & $4^{\text {th }}$ day & $80.2 \pm 3.9$ & $81.4 \pm 5.1$ & 0.326 \\
\hline & $5^{\text {th }}$ day & $80.2 \pm 3.9$ & $81.4 \pm 5.1$ & 0.326 \\
\hline \multicolumn{2}{|c|}{ P-value ${ }^{2}$} & \multicolumn{3}{|c|}{$(0.000 * *)$} \\
\hline \multirow{5}{*}{ : } & $1^{\text {st }}$ day & $90,0 \pm 0.50$ & $95.3 \pm 0.74$ &., 470 \\
\hline & $2^{\text {nd }}$ day & $94.7 \pm 1.02$ & $94.2 \pm 2.02$ & $\cdot, r \cdot T$ \\
\hline & $3^{\text {rd }}$ day & $95.7 \pm 1.1$ & $95.1 \pm 1.2$ & $0.04 *$ \\
\hline & $4^{\text {th }}$ day & $96.8 \pm 0.59$ & $94.1 \pm 1.5$ & $0.001 * *$ \\
\hline & $5^{\text {th }}$ day & $96.8 \pm 0.59$ & $93.4 \pm 1.6$ & $0.000^{* *}$ \\
\hline \multicolumn{2}{|c|}{ P-value $^{2}$} & \multicolumn{3}{|c|}{$0.000 * *)($} \\
\hline
\end{tabular}

\section{P-value1: $t$ test P-value2: Anova $\quad\left(1^{\text {st }}\right.$ day: Pre intervention $) \quad\left(2^{\text {nd }}\right.$ to $5^{\text {th }}$ day: Post intervention $)$}

* Statistical significant $(P \leq \mathbf{0 . 0 5}) * *$ highly Statistical significant $(P \leq 0.01)$

Table (3): Displayed that, there were highly statistical significant differences between both groups regarding to temperature during $4^{\text {th }}$ and $5^{\text {th }}$ day post intervention documented by $\mathrm{p}$ value $(0.004$ and 0.006$)$ respectively. The table reflected that, there were high statistical significant differences among both groups regarding to the respiration during $2^{\text {nd }}$ day post intervention documented by $\mathrm{p}$ value $(0.001)$. Also there were statistical significant differences among both groups regarding the respiration during $3^{\text {rd }}, 4^{\text {th }}$ and $5^{\text {th }}$ day post intervention documented by $p$ value $(0.049,0.05$ and 0.029$)$ respectively.

As regard to pulse there were statistical significant differences among both groups during $2^{\text {nd }}, 3^{\text {rd }}, 4^{\text {th }}$, and $5^{\text {th }}$ day post intervention documented by $\mathrm{p}$ value $(0.013,0.029,0.021$ and 0.024$)$ respectively. As regard to the systolic and diastolic blood pressure there were no statistical significant differences among both groups along the $1^{\text {st }}$ five days post intervention.

From the same table it was noticed that there were statistical significant differences among both groups regarding oxygen saturation during $3^{\text {rd }}$ day post intervention documented by p value (0.04), moreover, there were high statistical significant differences among both groups during $4^{\text {th }}$ and $5^{\text {th }}$ day post intervention documented by $\mathrm{p}$ value $(0.001$ and 0.000$)$ respectively.

Table (4): Comparison between the Study and Control Groups Regarding Effect of Cardiac Rehabilitation Exercise Protocol on Reducing Postoperative Cardiopulmonary Complications

\begin{tabular}{|c|c|c|c|c|c|c|}
\hline \multirow{2}{*}{ Variables } & \multicolumn{2}{|c|}{ Study $(n=30)$} & \multicolumn{2}{|c|}{ Control $(n=30)$} & \multirow{2}{*}{$\mathbf{X}^{2}$} & \multirow{2}{*}{ P-value } \\
\hline & No. & $\%$ & No. & $\%$ & & \\
\hline Cardiac & & & & & & \\
\hline Impaired Cardiac Output syndrome (ICOS) & 6 & 20 & 9 & 30 & \multirow[b]{2}{*}{7.2} & \multirow[b]{2}{*}{$0.027 *$} \\
\hline Ventricular Tachycardia (VT) & 3 & 10 & 5 & 16.7 & & \\
\hline Respiratory & & & & & & \\
\hline Respiratory Tract Infection (RTI) & 1 & 3.3 & 6 & 20 & \multirow{2}{*}{6.5} & \multirow{2}{*}{$0.038 *$} \\
\hline Plural effusion & 0 & 0 & 2 & 6.7 & & \\
\hline
\end{tabular}

* Statistical Significant Difference $(P \leq 0.05)$ 
Table (4): Showed that, (20\%) of study group have ICO syndrome as a cardiac complication early before discharge, compared to $(30 \%)$ of control group before discharge, There were statistical significant differences among both groups regarding cardiac complication during early post-operative reflected by $\mathrm{P}$-value $=0.027$

Moreover; it was found that, $(3.3 \%)$ of study group had RTI as a respiratory complication compared to (20\%) of control group. There were statistical significant differences among both groups regarding respiratory complication during early post discharge reflected by P-value $=0.038$

Table (5): Correlation between Age, BMI, Chronic Illness, \& Smoking and Occurrence of Cardiopulmonary Complications:

\begin{tabular}{|c|c|c|c|c|c|c|c|c|}
\hline \multirow{3}{*}{ Variables } & \multicolumn{4}{|c|}{ Cardiac Complications } & \multicolumn{4}{|c|}{ Respiratory Complications } \\
\hline & \multicolumn{2}{|l|}{ Study } & \multicolumn{2}{|c|}{ Control } & \multicolumn{2}{|l|}{ Study } & \multicolumn{2}{|c|}{ Control } \\
\hline & $\mathbf{r}$ & $\mathbf{P}$ & $\mathbf{R}$ & $\mathbf{P}$ & $\mathbf{R}$ & $\mathbf{P}$ & $\mathbf{r}$ & $\mathbf{P}$ \\
\hline Age & 0.067 & 0.725 & 0.30 & 0.106 & 0.370 & 0.44 & 0.247 & 0.188 \\
\hline BMI & 0.25 & 0.175 & 0.075 & 0.695 & 0.22 & 0.239 & 0.20 & 0.288 \\
\hline Chronic Illness & 0.030 & 0.873 & 0.076 & 0.691 & 0.125 & 0.512 & 0.313 & 0.092 \\
\hline Smoking & 0.021 & 0.913 & 0.025 & 0.879 & 0.125 & 0.526 & 0.25 & 0.183 \\
\hline
\end{tabular}

* Statistical Significant Difference $(\mathbf{P} \leq \mathbf{0 . 0 5})$

Table (5) showed that, there were no statistical significant correlation between occurrence of cardiac complications and age, BMI, chronic illness and smoking among study and control group as documented by $r$ value $(0.067 \& 0.30),(0.25 \& 0.075),(0.030 \&$ $0.076)$ and $(-0.021 \&-0.025)$ respectively.. As well as there were no statistical significant correlation between occurrence of respiratory complications and age, BMI, chronic illness and smoking among study and control group as documented by $\mathrm{r}$ value $(0.370$ $\& 0.247),(0.22 \& 0.20),(0.125 \& 0.313)$ and $(0.125 \& 0.25)$ respectively.

Table (6): Multiple Linear Regression Model Evaluating the Relationship between Cardiac Rehabilitation Exercise Protocol and Early Postoperative Complications:

\begin{tabular}{|l|c|c|c|}
\hline Early Postoperative Complications & \multicolumn{2}{|c|}{ Cardiac Rehabilitation Exercise Protocol } \\
\cline { 2 - 4 } & $\mathbf{B} \pm$ SE & F & 1.07 \\
\hline Respiratory & $-0.848 \pm 0.258$ & $0.042^{*}$ \\
\hline Cardiac & $-0.441 \pm 0.426$ & 10.84 & 0.479 \\
\hline
\end{tabular}

* Statistical Significant Difference $(\mathbf{P} \leq \mathbf{0 . 0 5})$

Table (6) Predicted that, the cardiac rehabilitation exercise protocol will decrease the occurrence of respiratory and cardiac complications, the regression model, shows the negative relation between cardiac rehabilitation exercise protocol and occurrence of respiratory and cardiac complications with significance to respiratory complications only as documented by $p$ value ( 0.042$)$.

\section{Discussion:}

As regard to socio-demographic characteristics and their medical data, The findings of the present study showed that there is no statistical significance difference between the two groups regarding to socio-demographic characteristics and their medical data, this homogeneous in terms of demographic, clinical, and surgical variables enabled the investigator to consider the impact observed in the intervention group as a consequent to performed cardiac rehabilitation exercise protocol. This results supported by (Thirapatarapong, \& Chumwong, 2017) who study the "pre-operative pulmonary training program in coronary artery bypass graft surgery patients at Siriraj hospital" and found that there was no significant difference between the two groups regarding the baseline characteristics such as age, sex and comorbidities.

The findings of present study showed that more than half of the total studied patients were in the age group from 30-50 years old of the study and control group; this is due to the selected sample which included the adult patient only, and that the highest percentages among study and control group were female, a possible explanation for this could be the fact that females are exposed to more cardiovascular risks in their lifetime than males this finding agrees with (Makalla, 2014), in study titled "the role of physiotherapy in the management of patients following cardiac surgery in Tanzania" and reported that nearly half of the total studied patients were in the age group from 28-52 and (Nyawawa, et al., 2010) study titled "one year experience of cardiac surgery at Muhimbili national hospital", who reported that $79 \%$ of all patients who had cardiac surgery were females.

This result is in contrast with (Falase, et al., 2013) a study titled "open heart surgery in Nigeria, a work in $P$ a g e | 51 progress", and reported that the patients received cardiac surgery aged from 2 years of age to 72 years of age and (Guo, \& Arthur, 2012) who investigated the effect of "a preoperative education intervention to reduce anxiety and improve recovery among Chinese cardiac patients" and reported that more than half of cardiac surgery patients were males.

As regard to the medical data among the studied subjects, the present study showed that the highest percentages of both study and control group were having rheumatic heart disease, the investigator rationalized that by the increased incidence of rheumatic heart disease in low and middle income countries and more than two third of both study and control groups had valve surgery, this is related to highest percentage of rheumatic heart disease among the studied sample affecting the heart valves and that that nearly half of both study and control group were suffering from hypertension and/or diabetes mellitus. Also, the highest percentages of both study and control group were nonsmoker with a normal body mass index.

The findings supported by (Musuku, et al., 2013) in a study titled "The synergies in open-heart surgery in Zambia" and reported that $(71.4 \%)$ of studied patients had rheumatic heart disease and (Gelijns, et al., 2014), who examined the "management practices and major infections after cardiac surgery" and found that the valvular surgery was the most common operation type. In addition to (Miranda, et al., 2011) in a study titled "evaluation of pain intensity and vital signs in the cardiac surgery postoperative period" and found that half of the studied population suffered from diabetes mellitus (DM) and/or systemic hypertension.

As regard to level of performance of the implemented cardiac rehabilitation exercise protocol, the Ahmed A., et al 
findings of the present study showed, that the highest percentage of the study group patient's possessed a satisfactory score regarding level of performance post implementing the cardiac rehabilitation exercise protocol. This finding supported by (Fatima, et al., 2017) who investigated the "pre and post-operative physiotherapy for patients after open-heart surgery" and demonstrated that the respondents to pre-operative information regarding, deep breathing, mobilization, supported coughing, range of motion exercises and post-operative sternotomy restrictions was $95 \%$ of all patients.

As regard to the hemodynamic variables among the studied subjects, the present study findings showed that the mean values of the vital signs and oxygen saturation measurements among the studied subjects were within the normal range, with statistical significant improvement in the study group, these variations due to the positive effect of implemented cardiac rehabilitation exercise protocol. This findings supported by (Ghashghaei, et al., 2012) who investigated the effect of "exercise-based cardiac rehabilitation in improving hemodynamic responses after coronary artery bypass graft surgery" and found that cardiac rehabilitation program have a significant effects on hemodynamic responses.

In the present study it has been noticed that a statistical significant differences were found among both groups regarding pulse and respiration during $2^{\text {nd }}, 3^{\text {rd }}, 4^{\text {th }}$ and $5^{\text {th }}$ day post intervention. This results supported by (Elgebaly, 2017) study titled " Does bilevel positive airway pressure improve outcome of acute respiratory failure after open-heart surgery?" who confirmed that there was statistical significant difference between the two groups in relation to heart rate and respiration. This result is in contrast with, (Ahmed, et al., 2015) who mentioned that there was no statistical significant difference was found between the studied groups during the postoperative period related to body heart rate and respiratory rate.

The present study findings displayed a statistical significant difference between both groups regarding to temperature during $4^{\text {th }}$ and $5^{\text {th }}$ day post intervention, the investigator regarded that to the effect of the implemented chest physiotherapy in reducing the respiratory tract infection in the study group. This result is in contrast with (Stiller, et al., 2010) who evaluate the "efficacy of breathing and coughing exercises in the prevention of pulmonary complications after coronary artery surgery" and reported that no significant differences between studied groups as regard to temperature at any stage of treatment during postoperative days.

The present study findings displayed that the mean value of systolic and diastolic blood pressure among the studied subjects were within the normal range, with no statistical significant differences among both groups along the $1^{\text {st }}$ five days post intervention, this could be due to the effect of the inotropic agents that is routinely administered post cardiac surgery. This result is in contrast with (Ghashghaei, et al., 2012) who found that cardiac rehabilitation has a significant effect on resting and maximum systolic blood pressure and resting and maximum diastolic blood pressure

The present study findings displayed a statistical significant difference between both groups regarding to peripheral oxygen saturation $\left(\mathrm{SpO}_{2}\right)$ during $3^{\text {rd }}, 4^{\text {th }}$ and $5^{\text {th }}$ day post intervention, the investigator regarded that to the effect of the implemented chest physiotherapy (deep breathing, coughing exercises and incentive spirometer). This result is supported by (Tariq, et al., 2017) a study titled "Postoperative outcome of early $\leq 3$ Mets (metabolic equivalent of tasks) of physical activity on patients outcomes after cardiac surgery" who documented that early physical activity led to marked improvement in oxygen saturation in the experimental group.

The findings of present study showed that the highest percentage of complications appeared in the $1^{\text {st }}$ five days postoperatively. These results were in agreement with (Winkelmann, et al., 2015) in study titled "analysis of steps adapted protocol in cardiac rehabilitation in the hospital phase" and demonstrated that the highest number of complications is on the $1^{\text {st }}$ three postoperative days, and the most common complications were respiratory, hemodynamic and cardiac conduction disorders.

In addition, it has been noticed that the total postoperative pulmonary complications were significantly lower among group who received pre-operative cardiac rehabilitation exercise training protocol. The investigator suggests that the pre-operative cardiac rehabilitation exercise training protocol could prevent the deterioration of lung capacity and weakness of the inspiratory muscle.

This result is in line with (Hulzebos, et al., 2012) in study titled "preoperative physical therapy for elective cardiac surgery patients" who indicated that a preoperative physical therapy mixed intervention including either breathing exercises or aerobic exercises and inspiratory muscle training decreases postoperative pulmonary complications and length of hospital stay in patients underwent elective cardiac surgery.

The present study findings showed that the study group had a statistical significance lower incidence of respiratory tract infection. This result is in harmony with (Thirapatarapong \& Chumwong, 2017) who evaluated the "pre-operative pulmonary training program in coronary artery bypass graft surgery patients at Siriraj hospital" and reported that the incidence of total pulmonary complications in study group was $5.58 \%$ and the most frequent pulmonary complication was pneumonia.

The previous result is in the same line with (Hulzebos, et al., 2012) who found that preoperative physical therapy including inspiratory muscle training, training and education of breathing and coughing techniques statistically significant decrease the post-operative pulmonary complications incidence as atelectasis and pneumonia.

Regarding the relations between the applied cardiac rehabilitation exercise training protocol and the presence of cardiac complications in the postoperative period, few studies were found on the same theme as the present study which was important to conduct discussions on the findings regarding this point.

As regard post cardiac complication the present study findings showed that impaired cardiac output syndrome and ventricular tachycardia were the most common post cardiac complications, this finding is supported by (Lomivorotov, 2017) a recent review of "Low-cardiac-output syndrome after cardiac surgery", who mentioned that lowcardiac-output syndrome (LCOS) and cardiac arrhythmias is the most common and the most serious complications after cardiac surgery and associated with increased morbidity, mortality, and healthcare resource utilization.

The present study findings showed that the study group has a significant decrease regarding cardiac complications, this result is in line with (Mungovan, et al., 2017) who study the "effect of physical activity in the first 
five days after cardiac surgery" and demonstrated that postoperative physical activity and prescribed exercise programs reduces postoperative complications, e.g. venous thromboembolism and pulmonary complications, improves postoperative physiological functional capacity. In addition, early participation in exercise programs is an integral component of rapid recovery leading to optimal surgical outcomes.

The finding presented that, there was positive correlation between respiratory complications occurrence and age, this agrees with (Friedrich, et al., 2009) who investigated the relation between cardiac surgery complications and elderly patient; the study found that the complication rate after cardiac surgical procedures in elderly patients is notably higher than in younger patients.

The present study findings showed that, there were positive correlation between postoperative respiratory complications occurrence and body mass index, this finding was supported by (Sabzi \& Faraji, 2016) who evaluated the effect of body mass index on postoperative complications in beating coronary artery surgery and found that high body mass index caused postoperative atelectasis and infection as increased body mass index tended to prolong the intubation time and cause respiratory muscle weakness and its consequent complications such as pulmonary infection.

The present study revealed a statistically positive correlation between occurrence of postoperative cardiac complications and body mass index; this was supported by (Gao, et al., 2016) who investigated the impact of body mass index on outcomes in cardiac surgery, he found a significant increase in the incidence of postoperative atrial fibrillation/flutter in obese patients.

In addition to, (Valkenet, et al., 2011) a systemic review of the effects of preoperative exercise therapy on postoperative outcome and mentioned that risk factors, such as obesity and diabetes, have been shown to be significantly related to postoperative surgical complications. This result is in contrast with (Sabzi \& Faraji, 2016) who found that body mass index had no impact on postoperative cardiac complications.

The findings displayed a positive correlation between the presence of chronic illness and occurrence of postoperative cardiac complications and respiratory complications. This result is in line with (Clough et al., 2012) who study the effect of co-morbid illness on mortality outcomes in cardiac surgery and demonstrated that the presence of comorbid conditions was as follows: hypertension, diabetes, severe obesity, vascular disease, chronic obstructive pulmonary disease were significant predictors of post cardiac surgery complications and inhospital mortality

Finally, the current study regression model predicted that; applying the cardiac rehabilitation exercise protocol in the future would decreases the occurrence of respiratory and cardiac complications with significance difference only with respiratory complications, the investigator interpreted that as the cardiac rehabilitation exercise protocol only can reduce respiratory complications but cardiac complications is affected by other factors as drug regimen and other treatment modalities (cardio- version for treating arrhythmia).

This finding in line with (Kashmiri, et al., 2012) who examined the incidence of complications after cardiac surgery and documented that, in cardiovascular complications, patients who went in to low cardiac output syndrome or cardiac arrhythmia (Atrial fibrillation or Ventricular tachycardia/ Ventricular fibrillation), number of patients requiring inotropes for more than 24 hours, Pace maker requirement, Defibrillated after surgery, use of Intra-Aortic Balloon Pump (IABP), pericardial effusion requiring aspiration was noted.

In addition to, (Lighthall \& Olejniczak, 2015) who investigated the routine postoperative care of patients undergoing coronary artery bypass grafting and mentioned that most of postoperative arrhythmias are self-limited and return to preoperative rhythm in a few weeks or less and that physical activity is generally recommended for complication prevention and quality of life improvement after cardiac surgery.

Also, further validation by (Mundada \& Retharekar, 2016) who evaluated the effects of postoperative cardiopulmonary rehabilitation program on inspiratory muscle strength and its potential association with improved functional capacity after coronary artery bypass graft surgery. The study concluded that cardiopulmonary rehabilitation is an important determinant of functional capacity and cardiopulmonary outcomes after coronary artery bypass graft surgery.

\section{Conclusion}

Based on current study findings, it can be concluded that the cardiac rehabilitation exercise protocol in the form of deep breathing and coughing, incentive spirometer, early ambulation and ROM induce significant improvement in reducing the risk of post-operative cardio pulmonary complications.

\section{Recommendations:}

- It must be stressed that the exercise training from the preoperative stage until hospital discharge is individualized for each patient.

- Hospitals should recommend implementing cardiac rehabilitation exercise protocols as a routine hospital policy among all cardiac surgical patients in all age groups

\section{Acknowledgment}

The investigator would like to acknowledge the contribution of all participants who kindly agreed to take part in the study. They generously gave their time and attention to conduct this study. This study would have been impossible without their generosity.

\section{References}

1) Adugbire, B. A., Aziato, L., \& Dedey, F. (2017). Patients' experiences of pre and intra operative nursing care in Ghana: A qualitative study. International Journal of Africa Nursing Sciences, 6, 45-51.

2) Ahmed, A. M., Eldeen, S. M. A., Youssef, H. A., Mohammed M. A. (2015). Comparison the effect of conservative therapy and blow bottle among open heart surgery patients for the prevention of postoperative pulmonary complications, e-ISSN: 2320-1959.p- ISSN: 2320-1940, 4(4), PP 42-55

3) Al-Qubati, F. A. A., Damag, A., \& Noman, T. (2013). Incidence and outcome of pulmonary complications after open cardiac surgery, Thowra Hospital, Cardiac center, Sana'a, Yemen. Egyptian 
Journal of Chest Diseases and Tuberculosis, 62(4), 775-780.

4) Clough, R. A., Leavitt, B. J., Morton, J. R., Plume, S. K., Hernandez, F., Nugent, W., ... \& O'connor, G. T. (2012). The effect of comorbid illness on mortality outcomes in cardiac surgery. Archives of Surgery, 137(4), 428-433.

5) Cordeiro, A. L. L., Borges, D. L., Peruna, M. P., Guimarães, A. R., \& Cacau, L. D. A. (2017). Correlation between Length of Hospital Stay and Gait Speed in Patients Submitted to Cardiac Surgery. International Journal of Cardiovascular Sciences, 30(2), 123-127.

6) Da Costa Torres, D., dos Santos, P. M. R., Reis, H. J. L., Paisani, D. M., \& Chiavegato, L. D. (2016). Effectiveness of an early mobilization program on functional capacity after coronary artery bypass surgery: A randomized controlled trial protocol. SAGE open medicine, 4, 2050312116682256.

7) Elgebaly, A. S. (2017). Does bilevel positive airway pressure improve outcome of acute respiratory failure after open-heart surgery?. Annals of cardiac anaesthesia, 20(4), 416.

8) Enger TB. (2017). risk factors for short and long term complications following adult cardiac surgery, doctoral theses at NTNU, (89); 13:16

9) Ettema, R. G., Van Koeven, H., Peelen, L. M., Kalkman, C. J., \& Schuurmans, M. J. (2014). Preadmission interventions to prevent postoperative complications in older cardiac surgery patients: a systematic review. International journal of nursing studies, 51(2), 251-260.

10) Falase, B., Sanusi, M., Majekodunmi, A., Animasahun, B., Ajose, I., Idowu, A., \& Oke, A. (2013). Open heart surgery in Nigeria; a work in progress. Journal of cardiothoracic surgery, 8(1), 615.

11) Fatima, R., Kazmi, S. A. M., Naqvi, S. I. H., \& Rizvi, A. S. (2017). Pre and post-operative physiotherapy for patients after open-heart surgery. 6 (2), European International Journal of Science and Technology

12) Friedrich, I., Simm, A., Kötting, J., Thölen, F., Fischer, B., \& Silber, R. E. (2009). Cardiac surgery in the elderly patient. Deutsches Ärzteblatt international, 106(25), 416.

13) Gao, M., Sun, J., Young, N., Boyd, D., Atkins, Z., Li, Z., ... \& Liu, H. (2016). Impact of body mass index on outcomes in cardiac surgery. Journal of cardiothoracic and vascular anesthesia, 30(5), 13081316.

14) García, S. H., Lago, E. P., Oquendo, J. Á. M., \& Rivas Estany, E. (2014). Hospitalization phase of cardiac rehabilitation. Protocol for cardiac surgery. CorSalud (Revista de Enfermedades Cardiovasculares), 6(3), 246-256.

15) Gelijns, A. C., Moskowitz, A. J., Acker, M. A., Argenziano, M., Geller, N. L., Puskas, J. D., ... \& Miller, M. A. (2014). Management practices and major infections after cardiac surgery. Journal of the American College of Cardiology, 64(4), 372-381.

16) Ghashghaei, F. E., Sadeghi, M., Marandi, S. M., \& Ghashghaei, S. E. (2012). Exercise-based cardiac rehabilitation improves hemodynamic responses after coronary artery bypass graft surgery. ARYA atherosclerosis, 7(4), 151.

17) Guo, P., East, L., \& Arthur, A. (2012). A preoperative education intervention to reduce anxiety and improve recovery among Chinese cardiac patients: a randomized controlled trial. International Journal of Nursing Studies, 49(2), 129-137.

18) Højskov, I. E., Moons, P., Hansen, N. V., La Cour, S., Olsen, P. S., Gluud, C., ... \& Berg, S. K. (2017). SheppHeartCABG trial-comprehensive early rehabilitation after coronary artery bypass grafting: a protocol for a randomised clinical trial. BMJ open, 7(1), e013038.

19) Hulzebos, E. H., Smit, Y., Helders, P. P., \& van Meeteren, N. L. (2012). Preoperative physical therapy for elective cardiac surgery patients. Cochrane Database Syst Rev, 11: :CD010118.

20) Kashmiri, Z. A., Dar, M. I., Idrees, F., Khan, A. Q., \& Hussain, A. (2012). Incidence of Complications after Cardiac Surgery, Single Unit Experience. Pakistan Heart Journal, 41(3-4).

21) Lewis, S. L., Dirksen, S.R., Heitkemper, M. M., Bucher, L., Harding, M. M. (2014). $9^{\text {th }}$ ed, Medicalsurgical nursing: assessment and management of clinical problems, cardiac surgery, chapter34, 758:763. Elsevier Health Sciences.

22) Lighthall, G. K., \& Olejniczak, M. (2015). Routine Postoperative Care of Patients Undergoing Coronary Artery Bypass Grafting on Cardiopulmonary Bypass. In Seminars in cardiothoracic and vascular anesthesia 19(2), pp. 78-86. Sage CA: Los Angeles, CA: SAGE Publications.

23) Lomivorotov, V. V., Efremov, S. M., Kirov, M. Y., Fominskiy, E. V., \& Karaskov, A. M. (2017). Lowcardiac-output syndrome after cardiac surgery. Journal of cardiothoracic and vascular anesthesia, 31(1), 291-308.

24) Makalla, A. R. (2014). The role of physiotherapy in the management of patients following cardiac surgery in Tanzania.

25) Miranda, A. D. F. A., Silva, L. D. F. D., Caetano, J. Á., Sousa, A. C. D., \& Almeida, P. C. (2011). Evaluation of pain intensity and vital signs in the cardiac surgery postoperative period. Revista da Escola de Enfermagem da USP, 45(2), 327-333.

26) Mundada, N., \& Retharekar, S. (2016). Effect of Respiratory Muscle Training As an Adjunct to Conventional Therapy in Phase I Cardiac Rehabilitation for Median Sternotomy Patients. International Journal of Therapies and Rehabilitation Research, 5(4), 224-233.

27) Mungovan, S. F., Singh, P., Gass, G. C., Smart, N. A., \& Hirschhorn, A. D. (2017). Effect of physical activity in the first five days after cardiac surgery. Journal of rehabilitation medicine, 49(1), 71-77.

28) Musuku, J., Bvulani, B., Makasa, E., Mwandila, M., Singh, H., Mbewe, W., Kalininchecho, S., Kavindele, D., Mulendele, E., \& Kangwa, C. (2013). Synergies in open-heart surgery in zambia in 2011. In 6th World Congress: Paediatric Cardiology and Cardiac Surgery, (p.5). Cape Town, South Africa: Cardiovascular journal of Africa, 24(1)

29) Naveed, A., Azam, H., Murtaza, H. G., Ahmad, R. A., \& Baig, M. A. R. (2017). Incidence and risk 
factors of Pulmonary Complications after Cardiopulmonary bypass. Pakistan journal of medical sciences, 33(4), 993.

30) Nichols, S., Nation, F., Goodman, T., Clark, A. L., Carroll, S., \& Ingle, L. (2018). CARE CRCardiovascular and cardiorespiratory Adaptations to Routine Exercise-based Cardiac Rehabilitation: a study protocol for a community-based controlled study with criterion methods. BMJ open, 8(1), e019216.

31) Nyawawa, E. T. M., Ussiri, E. V., Chillo, P., Waane, T., Lugazia, E., Mpoki, U., Luchemba, R., Wandwi, B., Nyangasa, B., Bgoya, J., \& Mahalu, W. (2010). Cardiac Surgery: One Year Experience of Cardiac Surgery at Muhimbili National Hospital, Dar es Salaam- Tanzania. East and Central African Journal of Surgery, 15(1), 111-118.

32) Sabzi, F., \& Faraji, R. (2016). Effect of Body Mass Index on Postoperative Complications in Beating Coronary Artery Surgery. Ethiopian journal of health sciences, 26(6), 509-516.

33) Seo, Y. G., Jang, M. J., Park, W. H., Hong, K. P., \& Sung, J. (2017). Inpatient cardiac rehabilitation programs' exercise therapy for patients undergoing cardiac surgery: National Korean Questionnaire Survey. Journal of exercise rehabilitation, 13(1), 76.

34) Snowdon, D., Haines, T. P., \& Skinner, E. H. (2014). Preoperative intervention reduces postoperative pulmonary complications but not length of stay in cardiac surgical patients: a systematic review. Journal of physiotherapy, 60(2), 66-77.
35) Stephens, R. S., \& Whitman, G. J. (2015). Postoperative critical care of the adult cardiac surgical patient. Part I: routine postoperative care. Critical care medicine, 43(7), 1477-1497.

36) Stiller, k., Montarello, J., Walace, M., Daff, M., Grant, R., Jenkins, S., Hall, B., Yates, H. \& Nurs, B. (2010). Efficacy of breathing and coughing exercises in the prevention of pulmonary complications after coronary artery surgery $105: 741-47$.

37) Tariq, M. I, Khan, A. A., Khalid, Z., Farheen, H., Siddiqi, F. A. \& Amjad, I., (2017). "Postoperative outcome of early $\leq 3$ Mets (metabolic equivalent of tasks) of physical activity on patients outcomes after cardiac surgery, Journal of the College of Physicians and Surgeons Pakistan, Vol. 27 (8): 490-494

38) Thirapatarapong, W., \& Chumwong, N. (2017). Preoperative Pulmonary Training Program in Coronary Artery Bypass Graft Surgery Patients at Siriraj Hospital. Siriraj Medical Journal, 62(4), 161-164.

39) Valkenet, K., van de Port, I. G., Dronkers, J. J., de Vries, W. R., Lindeman, E., \& Backx, F. J. (2011). The effects of preoperative exercise therapy on postoperative outcome: a systematic review. Clinical rehabilitation, 25(2), 99-111.

40) Winkelmann, E. R., Dallazen, F., Bronzatti, A. B. S., Lorenzoni, J. C. W., \& Windmöller, P. (2015). Analysis of steps adapted protocol in cardiac rehabilitation in the hospital phase. Brazilian Journal of Cardiovascular Surgery, 30(1), 40-48 